\title{
Tecnologia, Organizações e Produtividade: Lições do Paradoxo de Solow
}

\author{
Technology, Organizations and Productivity: \\ Lessons from Solow's Paradox
}

FRANCISCO LIMA CRUZ TEIXEIRA*,**

RESUMO: O objetivo deste trabalho é discutir a relação entre difusão de tecnologia, mudanças organizacionais e crescimento da produtividade. A literatura teórica e empírica sobre a relação entre difusão de tecnologia da informação (TI) e crescimento da produtividade é revisada, com foco no Paradoxo de Solow. É adotada uma abordagem que tenta explicar esse paradoxo sob a perspectiva da difusão de inovações tecnológicas e organizacionais. Nas conclusões, são apresentadas considerações sobre políticas públicas para lidar com as novas tecnologias.

PALAVRAS-CHAVE: Crescimento econômico; produtividade; tecnologia; mudança estrutural.

ABSTRACT: The objective of this work is to discuss the relationship between diffusion of technology, organizational changes and productivity growth. The theoretical and empirical literature on the relationship between information technology (IT) diffusion and productivity growth is reviewed, focusing on Solow's Paradox. An approach that tries to explain this Paradox from the perspective of the diffusion of technological and organizational innovations is adopted. In the conclusions, considerations about public policies for dealing with the new technologies are presented.

KEYWORDS: Economic growth; productivity; technology; structural change.

JEL Classification: O41; O10.

\footnotetext{
* Professor e pesquisador do Núcleo de Pós-Graduação em Administração da Universidade Federal da Bahia - UFBA, Salvador/BA, Brasil. E-mail: teixeira@ufba.br.

$* *$ O presente trabalho foi realizado com o apoio do CNPq, uma entidade do governo brasileiro voltada ao desenvolvimento científico e tecnológico.
} 


\section{INTRODUÇÃO}

Desde que, em 1987, Robert Solow, Prêmio Nobel de Economia, cunhou a famosa frase "Vêm-se computadores em toda parte, menos nas estatísticas de produtividade", ${ }^{1}$ que o chamado Paradoxo de Solow passou a ser um tema de pesquisa bastante concorrido nos Estados Unidos e na Europa. Pesquisas sobre o Paradoxo de Solow enquadram-se, porém, em uma linha mais ampla, perseguida pelos economistas desde a década de setenta, que tenta entender e explicar a vertiginosa queda no crescimento da produtividade nos países desenvolvidos, a partir do início daquela década. De fato, os dados apresentados na Tabela 1 não deixam dúvidas sobre a descontinuidade de um padrão de crescimento que vinha desde o pós-guerra e que era interpretado como a causa fundamental para a recuperação fenomenal das economias capitalistas, após aquele conflito. É importante lembrar que, desde o trabalho seminal de Solow (1956), os economistas que trabalham com a função de produção agregada da tradição neoclássica imputam ao famoso residual ${ }^{2}$ boa parte do desenvolvimento econômico.

O Paradoxo de Solow, no entanto, adiciona um novo enigma ao problema. Ora, se a produtividade é a medida fundamental da contribuição da tecnologia para a expansão econômica, como ela pode declinar justamente durante um período em que a mudança tecnológica, capitaneada pela microeletrônica, havia, aparentemente, se acelerado? Independentemente da resposta que possa ser dada, a consequência desse fenômeno, acreditam os economistas, é que, desde a metade dos anos setenta, o aumento do desemprego na Europa e das desigualdades salariais nos EUA estariam correlacionados com o declínio no crescimento da produtividade (ver, por exemplo, Baily e Chakrabarti, 1988).

O presente artigo tem por objetivo discutir a relação entre difusão de tecnologia, mudanças organizacionais e crescimento da produtividade. Para tanto, apresenta uma revisão bibliográfica da teoria e de estudos empíricos sobre a relação entre difusão da tecnologia da informação (IT) e crescimento da produtividade, com destaque para o Paradoxo de Solow. Adota uma abordagem que procura explicar o Paradoxo a partir dos processos de difusão e aprendizagem das inovações tecnológicas e organizacionais. Por último, apresenta, nas conclusões, observações a respeito de políticas públicas frente às novas tecnologias.

\footnotetext{
${ }^{1}$ Em inglês: "We see computers everywhere except in the productivity statistics". Essa frase consta de um artigo publicado no New York Times Book Review, de 12 de julho de 1987, denominado "We'd Better Watch Out”.

${ }^{2} \mathrm{O}$ residual, descoberto inicialmente pelo próprio Solow (1956), refere-se à parcela do crescimento econômico que não pode ser explicado por adições nos fatores da função de produção neo-clássica (trabalho e capital).
} 
Tabela 1: Crescimento da produtividade do setor privado

(percentual médio anual)

\begin{tabular}{lccc|ccc|ccc}
\hline Países & \multicolumn{3}{c|}{$\begin{array}{c}\text { Produtividade total } \\
\text { dos fatores }\end{array}$} & \multicolumn{3}{c|}{$\begin{array}{c}\text { Produtividade } \\
\text { do trabalho }\end{array}$} & \multicolumn{3}{c}{$\begin{array}{c}\text { Produtividade } \\
\text { do capital }\end{array}$} \\
\hline & Pré & $1974-$ & $1980-$ & $\begin{array}{r}\text { Pré } \\
\text { EUA }\end{array}$ & $1974-$ & $1980-$ & Pré & 1974 & 1980 \\
Japão & 1973 & 1979 & 1990 & 1973 & 1979 & 1990 & 1973 & 197 & 1990 \\
\hline Alemanha & 1,5 & $-0,4$ & 0,2 & 2,1 & 0,0 & 0,6 & 0,1 & $-1,3$ & $-0,7$ \\
França & 4,6 & 0,9 & 1,6 & 8,0 & 2,9 & 2,9 & $-3,0$ & $-3,5$ & $-1,4$ \\
Itália & 2,5 & 1,7 & 1,0 & 4,4 & 3,0 & 1,7 & $-1,4$ & $-1,0$ & $-0,5$ \\
Reino Unido & 3,8 & 1,6 & 1,5 & 5,3 & 2,9 & 2,4 & 0,9 & $-1,0$ & $-0,2$ \\
Canadá & 4,1 & 1,9 & 1,2 & 6,1 & 2,8 & 1,9 & 0,4 & 0,3 & $-0,1$ \\
Média ponderada & 2,5 & 0,5 & 1,6 & 3,6 & 1,5 & 2,1 & $-0,3$ & $-1,6$ & 0,4 \\
da OECD (1) & 2,0 & 0,8 & 0,1 & 2,8 & 1,5 & 1,2 & 0,6 & $-0,5$ & $-1,8$ \\
\hline
\end{tabular}

Fonte: OECD 119961

(1) Inclui, além dos países do G 7, listados na tabela, Áustria, Bélgica, Dinamarca, Finlândia, Grécia, Islândia , Irlanda, Países Baixos, Noruega, Portugal, Espanha, Suécia, Austrália e Nova Zelândia.

\section{AS EXPLICAÇÕES PARA O PARADOXO DE SOLOW}

Até agora, as explicações para o Paradoxo não são consensuais, nem definitivas. Elas podem ser agrupadas em quatro grandes categorias.

Em primeiro lugar, não se pode desprezar as variáveis macroeconômicas que impactam o aumento da produção e da produtividade. Os números mostram um declínio no crescimento da produtividade a partir do início dos anos setenta, em comparação com os vinte e cinco anos anteriores. O pós-guerra foi um período caracterizado pelo sucesso das políticas de cunho keynesianas, que asseguraram uma relativa estabilidade macroeconômica nos principais países capitalistas. A manutenção dessa estabilidade começa a apresentar problemas no fim dos anos sessenta. Porém, é a partir de 1973, com o advento de choques resultantes de aumentos rápidos e significativos nos preços relativos, notadamente de algumas commodities estratégicas, que o mundo ocidental entra em uma trajetória de turbulências. A década de oitenta é marcada por profundas flutuações financeiras e monetárias, em um quadro que muitos consideram de demanda saturada. O resultado é que declinam as taxas de investimento e de formação de capital humano, o que teria reduzido a capacidade das empresas de explorarem o potencial das novas tecnologias (Bell, Chesnais e Wienert, 1992). Contudo, mesmo considerando que a deterioração do ambiente econômico havia causado importantes efeitos sobre o crescimento da produtividade, não se pode relacionar o fenômeno apenas às variáveis de natureza macro, como argumenta Lindbeck (1992). Esse argumento torna-se mais forte quando se analisa o comportamento da produtividade nos anos noventa, época de relativa estabilidade e crescimento, o que será feito mais adiante.

Outra linha de interpretação do Paradoxo de Solow refere-se a questões de 
ordem quantitativa, perseguida por economistas que adotam o referencial neoclássico e que trabalham com funções de produção agregadas.

O primeiro argumento dentro dessa linha está relacionado com a ideia de convergência (catching-up), derivada da própria teoria de crescimento dos neoclássicos. Segundo esse raciocínio, existiriam oportunidades adicionais para o crescimento da produtividade quando um país está operando em níveis menores do que os registrados nos países líderes. Isso porque o processo de difusão de tecnologias, originárias dos países líderes, ofereceria oportunidades para a rápida introdução de novos sistemas produtivos, via renovação e expansão de capital, assim como para a modernização organizacional. Além disso, o processo de crescimento econômico, alimentado pelos novos investimentos e tecnologias, tende a elevar a produtividade dos trabalhadores ocupados em atividades de baixo desempenho, a exemplo da agricultura e pequeno comércio, através do aumento do emprego nas atividades modernas (Abramovitz, 1992).

De acordo com a ideia da convergência, o Paradoxo de Solow seria explicado pelo fato de o processo de catch-up dos países europeus e do Japão em relação aos Estados Unidos ter se esgotado. O problema com essa explicação é bastante claro: ela até poderia explicar o decréscimo no crescimento da produtividade nos outros países, mas não nos próprios EUA onde, como mostra a Tabela 1, foi bastante acentuado. Além disso, Baumol, Nelson e Wolff (1994) acentuam que, para os países mais prósperos, desde a metade da década de setenta, observa-se uma tendência à divergência no crescimento da produtividade, apesar do residual continuar convergindo.

O segundo argumento, dentro da linha quantitativa, levanta a possibilidade de existirem erros de mensuração dos índices de produtividade. A hipótese, levantada por Baily e Gordon (1988), por exemplo, é que a produção, o numerador dos cálculos de produtividade, não estaria sendo medida corretamente devido, principalmente, às dificuldades para desenvolver deflatores de preços precisos - em função do processo inflacionário errático - e ajustados às mudanças na qualidade e preços dos novos produtos.

Após extensivo trabalho empírico, Baily e Gordon (1988), utilizando dados agregados dos setores industriais e de serviços dos EUA, chegaram à conclusão de que, apesar de problemas de mensuração existirem, principalmente no setor de serviços, eles não são capazes de explicar o fenômeno do decréscimo nos índices de produtividade. O computador estaria sendo difundido com grande velocidade na economia americana e teria causado um impacto significativo na maneira como os negócios são conduzidos. Porém, os setores onde computadores e outros equipamentos eletrônicos estão sendo usados estariam experimentando importantes decréscimos no crescimento da produtividade. O trabalho de Gordon (1993) estende essas mesmas conclusões para todo o período de 1972 a 1991, tanto para produtividade do trabalho corno para a produtividade total dos fatores.

Ainda relacionado a problemas de mensuração, levanta-se o argumento da qualidade. A ideia é que os computadores e equipamentos relacionados poderiam estar oferecendo serviços de valor para os consumidores - melhor qualidade, va- 
riedade, serviços, agilidade e velocidade de resposta -, mas que não são mensurados pelas estatísticas oficiais. Isso porque os deflatores utilizados para medir os gastos dos consumidores seriam incapazes de incorporar mudanças na natureza dos produtos e serviços, tornadas possíveis pelo uso da tecnologia da informação (Brynjolfsson, 1993).

Existiriam três problemas com esse argumento. Primeiro, quando se mensura o decréscimo no crescimento da produtividade, compara-se o período iniciado no início dos anos setenta com aquele do pós-guerra. Ora, é de conhecimento geral que o imediato pós-guerra foi um período de intensas mudanças tecnológicas, decorrentes de um conjunto de inovações nas áreas da indústria química-petroquímica, eletroeletrônica e metal-mecânica (Freeman, 1992). Essas inovações também provocaram mudanças substanciais na qualidade dos produtos e serviços. Então, se os métodos de mensuração da produtividade não são adequados para as inovações decorrentes da tecnologia da informação, por que seriam adequados para as inovações do pós-guerra? Pressupondo a pertinência dessa questão, também poder-se-ia indagar até que ponto a difusão da tecnologia da informação conforma um período de intensa mudança tecnológica. Poucos duvidam, entretanto, do potencial revolucionário da IT. Tampouco os gastos em P\&D, principal indicador do esforço voltado para inovação tecnológica, revelam decréscimo nas últimas décadas (OECD, 1996).

O segundo problema relaciona-se ao conceito de qualidade que se tem em mente, quando se levanta o argumento. Hoje, é praticamente consenso que, do ponto de vista da competição por mercados, a qualidade de produtos e serviços evolui de acordo com as necessidades dos clientes e não pela definição técnica de novas especificações. Em sendo assim, os sistemas produtivos baseados em novas tecnologias (inclusive organizacionais) deveriam ser capazes de atender aos requisitos de mercado e aumentar a produtividade. Esse seria o caso, por exemplo, do sistema de produção da Toyota na indústria automobilística. A diversificação da produção, incluindo produtos de melhor qualidade, é uma estratégia para ganhar mercados. Se a estratégia funciona, ela deve aparecer no aumento da produção e, portanto, da produtividade.

O último problema diz respeito ao questionamento que se faz quanto à melhoria na qualidade dos produtos e serviços. Vários indicadores mostram que a qualidade dos serviços tem decrescido e não aumentado. Por outro lado, questionam-se as vantagens, do ponto de vista do consumidor, de se dispor de uma grande variedade de produtos irrelevantes, a exemplo de goma de mascar (Brynjolfsson, 1993). Além disso, para que esse argumento pudesse explicar o Paradoxo de Solow, haveria de se provar que as melhorias de qualidade atuais são mais significativas do que as do pós-guerra.

Uma outra abordagem ao problema da quantificação dos impactos da tecnologia da informação na produtividade é apresentada por Oliner e Sichel (1994). Trabalhando do ponto de vista da função de produção agregada neoclássica, os autores detectam a rápida difusão da tecnologia da informação, alimentada, em grande parte, pela queda vertiginosa dos preços, conforme mostra a Tabela 2. Duas 
observações sobre essa tabela são importantes. Primeiro, além do conceito de "participação real no estoque líquido de capital", utiliza-se o conceito de "a participação nominal no estoque líquido de capital”. De acordo com Oliner e Sichel (1994), o conceito de "participação nominal" não sofre os efeitos de não se computar a queda dramática nos preços dos equipamentos de IT, após o ano base escolhido para deflacionar os valores e, portanto, evita a superestimação do peso da IT nos investimentos e no estoque de capital. Segundo, a categoria "computadores e outros equipamentos de processamento de informações" engloba, além de computadores e periféricos, equipamentos de escritório e de contabilidade, equipamentos de comunicação, instrumentação científica e máquinas copiadoras. Essa categoria amplia bastante o conceito tradicional de IT, que considera apenas computadores e periféricos.

Com base em números que são, aparentemente, mais precisos, Oliner e Sichel (1994) chegam à conclusão de que o Paradoxo de Solow seria simplesmente uma falsa questão, uma vez que a contribuição da IT para o aumento da produtividade não poderia ser significativa, dado que a sua participação no estoque de capital produtivo é, ainda, muito pequena e não deverá crescer muito nos próximos anos.

Tabela 2: Participação de computadores e outros equipamentos de

processamento de informações no total de equipamentos e estruturas não residenciais (EUA, \%, 1970-93, ano base 1987)

\begin{tabular}{|c|c|c|c|c|c|c|}
\hline Equipamentos e medidas & 1970 & 1975 & 1980 & 1985 & 1990 & 1993 \\
\hline \multicolumn{7}{|l|}{ Computadores e periféricos } \\
\hline \multicolumn{7}{|l|}{ Participação real } \\
\hline - Investimentos & 0,1 & 0,2 & 1,0 & 4,7 & 8,7 & 17,8 \\
\hline - Estoque líquido de capital & 0,0 & 0,1 & 0,2 & 1,3 & 3,1 & 5,1 \\
\hline \multicolumn{7}{|l|}{ Participação nominal } \\
\hline - Investimentos & 2,6 & 2,1 & 3,5 & 6,4 & 6,3 & 7,6 \\
\hline - Estoque líquido de capital & 0,8 & 0,6 & 0,9 & 1,8 & 2,1 & 2,0 \\
\hline \multicolumn{7}{|l|}{$\begin{array}{l}\text { Participação nominal no estoque líquido } \\
\text { de capital }\end{array}$} \\
\hline $\begin{array}{l}\text { - Computadores e equipamentos de } \\
\text { escritório e de contabilidade e de } \\
\text { contabilidade }\end{array}$ & 1,6 & 1,3 & 1,5 & 2,0 & 2,2 & 2,0 \\
\hline $\begin{array}{l}\text { - Equipamentos de processamento de } \\
\text { informações }\end{array}$ & 7,1 & 7,1 & 7,6 & 10,1 & 11,1 & 11,7 \\
\hline
\end{tabular}

Fonte: Oliner e Sichel (1994)

Muito embora Oliner e Sichel (1994) apresentem uma persuasiva explicação para o Paradoxo, algumas objeções, no entanto, podem ser levantadas. Em primeiro lugar, mesmo expandindo os elementos que compõem a categoria IT - o que, por si, já aumenta consideravelmente a sua participação no estoque de capital -, os autores, declaradamente, não tentam quantificar os impactos daquilo que eles chamam de "revolução microeletrônica como um todo". Caso isso seja feito, a 
fatia da high-tech no estoque de capital pode chegar a $25 \%$, como mostrado em Roach (1991). Nesse caso, não se pode dizer que essa fatia é pequena para, potencialmente, ter um impacto tão insignificante no crescimento da produção. Por outro lado, considerando-se o peso da microeletrônica a partir dos setores consumidores de semicondutores, os dados apresentados por Flamm (1996) mostram que os setores listados na Tabela 2 representavam menos de $60 \%$ do mercado total de chips, em 1987.

Em segundo lugar, mesmo considerando apenas os elementos da categoria IT usados por Oliner e Sich el (1994), deve-se atentar para o fato de que o problema do baixo crescimento da produtividade é particularmente agudo no setor de serviços, onde a maioria dos equipamentos de IT são utilizados. Com efeito, até 1970, o crescimento da produtividade na área de serviços acompanhou o do setor manufatureiro, mas, desde então, as tendências vêm divergindo consideravelmente, como pode ser visto na Tabela 3. A questão é que, de acordo com Roach (1991), o setor de serviços possui mais de $85 \%$ da base instalada de IT, nos EUA, quando se considera as categorias usadas por Oliner e Sichel. Esses números levaram Roach a concluir que os investimentos massivos em IT não contribuíram para a melhoria da produtividade, pelo contrário, tornaram o setor de serviços americano menos lucrativo e menos competitivo.

Tabela 3: Crescimento médio anual da produtividade agregada. 1948-1993, períodos selecionados (EUA \% por ano)

\begin{tabular}{lccc}
\hline \multicolumn{1}{c}{ Medida } & $1948-73$ & $1973-87$ & $1987-93$ \\
\hline $\begin{array}{l}\text { Produtividade de trabalho } \\
\text { manufatura }\end{array}$ & 2,82 & 2,52 & 2,45 \\
$\quad$ serviços & 2,32 & 0,25 & 0,21 \\
$\begin{array}{l}\text { Produtividade total dos fatores } \\
\text { manufatura }\end{array}$ & & \\
$\quad$ serviços & 2,03 & 1,68 & 1,87 \\
\hline
\end{tabular}

Fonte: Baily e Gordon (1988 - 1948-87 - e Gordon (1993) - 1987-93.

A partir de 1993, começaram a surgir reportagens e estudos esperançosos quanto à reversão da tendência de baixo crescimento da produtividade dos anos setenta e oitenta. Com a estabilidade, principalmente nos EUA, os fatores macroeconômicos estariam controlados. Além disso, um movimento de reestruturação empresarial havia se formado e os investimentos em IT continuavam aumentando. Entretanto, analisando os dados até 1995, Oliner (1995) não encontrou evidências para sustentar o otimismo. Utilizando o método de "ponderação em cadeia", em lugar do método usual de "ponderação fixa "3, para o cálculo da produção, o autor

\footnotetext{
${ }^{3}$ Corresponde, em inglês, a "chain-weighted" e " fixed-weighted". O método do "fixed-weighted" utiliza um ano base (1987, no caso dos EUA) para deflacionar os preços, quando o PIB é calculado. $\mathrm{O}$ "chain weighted" calcula o crescimento do PIB, em um dado ano, através da média geométrica das mudanças produzidas por dois índices com ponderação fixa, que usam os preços do ano
} 
conclui que, de 1990 a 1995, o crescimento médio da produtividade do trabalho, nos EUA, foi de $1,3 \%$ e da produtividade total dos fatores de apenas $0,3 \%$. Utilizando o mesmo método para o período 1960-73, os números são 3,0 e 2,1\%, respectivamente. A "revolução microeletrônica", tão comentada por tantos autores, não estaria, ainda, provocando efeitos positivos significativos no crescimento da produtividade, pelo menos do ponto de vista dos economistas.

A polêmica em torno dos problemas quantitativos do crescimento da produtividade revela, entre outras coisas, as limitações da abordagem da função de produção neoclássica para lidar com o tema. Em primeiro lugar, existe uma questão técnica. Acredita-se que a produtividade total dos fatores é uma medida mais adequada para interpretar o Paradoxo do que a produtividade do trabalho. Porém, como comenta Triplett (1994), ao se utilizar a metodologia para cálculo do crescimento da produção, a produtividade total dos fatores se comporta como uma constante, pois é estimada a partir da produtividade do trabalho. Por outro lado, de acordo com Nelson (1996), não faz sentido tentar separar, quantitativamente, a contribuição de cada fator para o crescimento econômico e da produtividade, como se não houvesse interação e complementaridade entre eles. Pelo contrário, ele considera que as fontes de crescimento da produtividade - avanço tecnológico, formação de capital e melhoria da educação - devem ser vistas como ingredientes da receita de um bolo: todos são necessários, mas é impossível destacar a contribuição de cada um no resultado final.

Outro problema sério com a abordagem da função de produção diz respeito à sua própria utilidade para explicar um fenômeno como o Paradoxo de Solow. As medidas de produtividade agregadas, englobando toda a economia ou grandes setores, como o manufatureiro, apesar de indicarem tendências gerais, não são capazes de expressar o que acontece no lado real do problema: o nível microeconômico. O Paradoxo seria, em última instância, uma questão que diz respeito à difusão de tecnologia. A difusão é um processo que está vinculado a decisões gerenciais, informadas pelas circunstâncias particulares do ambiente competitivo e das restrições técnicas. Disso resulta que, no mundo real, existe uma enorme variedade na produtividade das empresas, das plantas, das células de produção e essa variedade não é captada - pelo contrário, é conceitualmente excluída da abordagem da função de produção neoclássica. Essa parece ser a opinião mesmo de economistas que trabalham com o paradigma neoclássico, como Mairesse e Grilliches (1990).

\section{DIFUSÃO E APRENDIZADO TECNOLÓGICO E ORGANIZACIONAL}

Uma outra abordagem para entender o Paradoxo de Solow é sugerida por David (1990). Esse autor compara a difusão da tecnologia da informação, no fim

corrente e do ano anterior como pesos. Acredita -se que o "chain-weighted" é mais adequado, pois consegue anular o efeito da queda dos preços dos bens de alta tecnologia. 
do século XX, com o que aconteceu cem anos antes com o motor elétrico. O seu argumento é que inovações tecnológicas básicas, como o dínamo e a IT, levam tempo para se difundir pela economia, em parte porque, de início, não é economicamente vantajoso substituir as velhas tecnologias. Além disso, para que haja um impacto importante na produtividade agregada, torna-se necessário que o processo de difusão esteja bastante adiantado: David estima que os efeitos do motor elétrico só se tornaram visíveis a partir de 1920, quando mais da metade das fábricas haviam sido eletrificadas.

Porém, mais importante do ponto de vista deste trabalho, é que, de acordo com David (1990), aplicações realmente revolucionárias das novas tecnologias requerem um período de aprendizagem que não foi, no caso do motor elétrico, curto. Essa aprendizagem diz respeito não apenas às questões técnicas das inovações, mas também às mudanças organizacionais necessárias para que elas possam impactar significativamente na produtividade dos fatores. No caso do motor elétrico, o período de difusão e aprendizagem durou quase quarenta anos e envolveu mudanças radicais nos meios utilizados para planejar e gerenciar as fábricas. Paul David considera a possibilidade de que, com a microeletrônica, esse período seja menor. Porém, ele considera também a possibilidade de que esteja havendo um forte componente inercial na evolução de organizações, no sentido de se adequarem a formas de produção intensivas em informação.

A hipótese de David (1990) direciona a tentativa de entender o Paradoxo de Solow para um outro caminho. Em lugar de procurar explicações com base em quantificações baseadas na função de produção agregada - que podem, no máximo, indicar a extensão do problema-, o estudo do processo de difusão e aprendizagem associados às novas tecnologias, a partir de variáveis organizacionais e institucionais, seria um caminho mais promissor. Nessa linha, a proposta de que a emergência da IT teria o potencial para conformar um novo "paradigma técnico econômico", poderia ser um ponto de partida. Esse conceito, de acordo com Bell, Chesnais e Wienert (1992), enfatiza as relações entre mudanças tecnológicas, organizacionais e institucionais no processo de desenvolvimento de um novo paradigma. A relação dessas mudanças com o crescimento da produtividade, no longo prazo, é, para Freeman (1992), muito clara. O potencial de crescimento da produtividade é, inicialmente, detectado em um número reduzido de setores. Daí, o processo de difusão se espalha por toda a economia. Mas, como esse processo exige mudanças nas instituições, organizações, infraestrutura, qualificações profissionais e equipamentos, existiria um inevitável período de adaptação estrutural (aprendizado). Portanto, a explicação de um fenômeno macroeconômico - crescimento da produtividade agregada - só seria possível a partir de variáveis microeconômicas - difusão de inovações nas empresas - em consonância com a hipótese Schumpteriana (Dosi, Giannetti e Toninelli, 1992).

A abordagem evolucionista (Nelson e Winter, 1982) privilegia, enquanto objeto de análise, o processo de difusão das novas tecnologias e o concomitante processo de aprendizagem, para que se possa entender o comportamento da produtividade. A visão tradicional do processo de difusão, que trabalha apenas com 
variáveis explicativas de natureza técnico-econômicas (taxa esperada de retorno, incertezas técnicas, etc.), não seria suficiente. A abordagem evolucionista entende a difusão como um processo muito mais complexo. Ela depende de estratégias empresarias, que são diferenciadas, e que levam a uma situação de permanente assimetria e heterogeneidade entre firmas competindo em mercados específicos. Retornos crescentes e dinâmicos são auferidos durante o processo, resultantes da aquisição de capacitações específicas, decorrentes do aprendizado (learning) que ocorre no interior das firmas.

O conceito de aprendizado, entendido enquanto uma série de mecanismos pelos quais as empresas geram as capacitações necessárias para operar, adaptar e modificar produtos, processos produtivos e estruturas organizacionais, tem sido enfatizado na literatura que trata de inovação tecnológica. Para Malerba (1992), por exemplo, o aprendizado é essencial para entender o aumento da produtividade que as firmas obtêm ao longo do processo de difusão de uma nova tecnologia. Ele estaria correlacionado com a possibilidade de compreensão dos sinais e símbolos do ambiente, visando incorporá-los ao processo produtivo e à sua organização. É importante observar que esse conceito de aprendizado diferencia-se da formulação inicial de Arrow (1962) que relacionava o crescimento da produtividade no interior da firma ao aprender fazendo (learning-by-doing). Nessa perspectiva, os ganhos da produtividade seriam resultantes apenas do tempo de produção e/ou da quantidade produzida. Porém, o aprendizado não se dá apenas by doing, mas através de estratégias empresariais que têm o objetivo explícito de aumentar a produtividade por meio de inovações, mesmo que incrementais e localizadas. Lundvall (1992) chama atenção para o fato de que o aprendizado corresponde a um cumulativo processo de aquisição e assimilação de conhecimento, que envolve, além das empresas, as instituições, e no qual o ambiente econômico desempenha papel fundamental.

O duplo e inter-relacionado processo de difusão e aprendizagem teria, portanto, o poder de modificar, paulatinamente, a inovação básica inicial, através da introdução de inovações secundárias, o que é fundamental para se entender os ganhos das empresas em termos de lucros e produtividade. Adaptações organizacionais desempenham papel fundamental no processo. Em resumo, como proposto por Nelson (1996), difusão é muito mais que a simples adoção de uma tecnologia. Ela é um processo evolutivo, que envolve o co-desenvolvimento de mercados, estilos organizacionais e inovações tecnológicas. A sua velocidade é determinada, conforme explica Dosi (1985), pela capacitação dos agentes produtores e receptores da inovação, pelos sinais econômicos emitidos pelo mercado e pela natureza das instituições que afetam tanto os sinais econômicos, como o processo de aprendizado.

A postulação da tese evolucionista parece que fornece suporte para a quarta, e última, categoria explicativa do Paradoxo de Solow. Como mostra Brynjolfsson (1993), vários autores sustentam que, com relação à firma, a IT não estaria trazendo os retornos esperados. De fato, Farbey, Targett e Land (1995) são enfáticos ao apontarem as limitações das análises tradicionais de custos e benefícios quando aplicadas a investimentos em IT. Mesmo assim, os investimentos são feitos porque os decisores estariam enfrentando dificuldades para quantificar os seus benefícios. 
Segundo Johnson (1992), muitas vezes as firmas não são capazes de estimar claramente as expectativas de lucros advindos de uma inovação, mas consideram a sua adoção uma necessidade para a sobrevivência. No caso da IT, uma vaga percepção da importância de se adquirir flexibilidade organizacional estaria por trás dos investimentos. De acordo com essa interpretação, a produtividade não estaria crescendo porque a capacidade gerencial ainda não é adequada aos requisitos organizacionais do novo paradigma técnico-econômico. Consequentemente, existiria uma diferença significativa entre o potencial para o incremento da produtividade e o que está sendo efetivamente obtido nas firmas e na indústria. Essa seria a base do Paradoxo de Solow, que se refletiria no nível agregado.

Neste ponto, cumpre levantar a evidência empírica disponível a respeito da relação entre investimentos em IT e ganhos de produtividade. A revisão dos estudos microeconômicos feita por Brynjolfsson (1993) apresenta um quadro contraditório. $\mathrm{Na}$ indústria manufatureira, estudo feito por Loveman (1994), por exemplo, apresenta as conclusões de uma pesquisa, que teve como base 60 unidades de negócios de empresas americanas e da Europa Ocidental. O resultado principal é que, para essa amostra, a IT teve pequeno, se é que teve algum, impacto marginal na produção ou produtividade do trabalho, enquanto todos os outros insumos, incluindo bens de capital tradicionais, tiveram impactos positivos e significativos na produção e na produtividade da mão-de-obra. Já estudo feito pelo próprio Brynjolfsson e Hitt (1993), com 380 grandes empresas, entre 1987 e 1991, usando essencialmente a mesma metodologia de Loveman (regressões de mínimos quadrados utilizando parâmetros da função de produção), encontrou resultados diferentes. O retorno sobre o investimento de capital em IT foi de $50 \%$ por ano e o retorno de gastos com pessoal de sistemas de informação havia sido também muito alto. O setor de serviços apresenta problemas mais agudos de mensuração dos resultados dos investimentos em IT do que o manufatureiro. Por isso, as conclusões dos estudos empíricos nesse setor são ainda mais contraditórias. Por exemplo, na amostra das empresas de serviços do estudo de Brynjolfsson e Hitt (1993), obteve-se um retorno de $60 \%$ por ano sobre os investimentos em IT. Já o trabalho de Roach (1991) mostra que, enquanto houve um vasto aumento do capital de IT por trabalhador, desde 1962 até 1989, a produção por trabalhador do setor de serviços decresceu, no mesmo período. Por outro lado, estudo qualitativo feito por Clemens (1991), com cinco empresas de diferentes sub-setores da área de serviços, revelou que os resultados dependem da estratégia utilizada nos investimentos em IT, assim como da natureza dos recursos e qualificações empregados.

O argumento de Brynjolfsson (1993), de que retornos positivos de investimentos em IT são encontrados na medida em que os estudos são mais recentes e as bases de dados mais amplas e confiáveis, parece que não é confirmado por trabalho lançado recentemente por Strassman (1997). Em pesquisa com 183 firmas em vários setores, o autor concluiu que empresas com os maiores percentuais dos orçamentos dedicados à IT têm mais do dobro de possibilidades de estarem entre as menos produtivas da amostra. As com menores orçamentos têm o dobro de possibilidades de estarem entre as mais produtivas. Embora não sejam evidências defi- 
nitivas, esse trabalho reacende o debate em torno dos benefícios empresariais advindos dos investimentos em IT. De qualquer forma, por enquanto, não se pode afirmar que existe uma relação clara entre investimentos em IT e desempenho das empresas (Galliers, 1995).

Quando o processo de difusão de novas tecnologias é analisado a partir de uma perspectiva evolucionista, outras variáveis, diferentes do crescimento da produtividade da firma, devem ser levadas em consideração. A introdução de uma nova tecnologia por uma empresa objetiva, em última instância, elevar a sua competitividade e lucratividade, em relação aos concorrentes. Isso pode acontecer sem que haja um correspondente aumento de produtividade, quando mensurada pelos parâmetros tradicionais da função de produção. Assim, a tecnologia da informação deveria ser analisada enquanto arma competitiva, capaz de diferenciar e elevar a qualidade dos produtos oferecidos, redividir mercados e, portanto, mudar a estrutura de setores específicos. Isso significa dizer que, no processo de difusão da IT, haverá firmas, setores, regiões com maior capacidade de incorporá-la às suas estratégias e, ao mesmo tempo, deslanchar um processo de aprendizado diferenciado, tornando-se mais competitivas em relação aos concorrentes. Com efeito, pesquisa realizada sobre o impacto da $1 \mathrm{~T}$ no setor de serviços dos EUA concluiu que os maiores benefícios não são detectados por medidas convencionais de produtividade, mas por melhorias no desempenho das empresas e suas atividades (National Research Council, 1994). Contudo, é importante observar que, do ponto de vista do Paradoxo, a melhoria de desempenho de uma empresa pode se dar em detrimento de outra. Dessa forma, o mercado poderia estar sendo apenas redividido, e não ampliado, gerando impactos insignificantes na produtividade agregada.

Para muitos autores (Adler, 1992, por exemplo), os benefícios da introdução de uma tecnologia tão revolucionária como a IT só se tornam significativos quando ela é acompanhada por mudanças organizacionais. Triplett (1994), por exemplo, lembra que o uso do motor elétrico em novas aplicações foi precedido de mudanças significativas nos velhos métodos de produção. Já no caso da IT, as mudanças organizacionais estão sendo feitas após a introdução dos equipamentos. Em sendo assim, o aproveitamento dos benefícios potenciais não estaria sendo prontamente realizado, adiando seus efeitos sobre os índices de produtividade. Isso seria explicado pelas características da própria tecnologia de base microeletrônica: ela possui uma natureza aberta e passível de constantes inovações e adaptações, quando submetida a um dinâmico processo de aprendizado (Ciborra e Schneider, 1992).

A relação entre automação de base microeletrônica e mudanças organizacionais é um tema bastante explorado na literatura. A ideia prevalecente é que para uma empresa ser capaz de usar a IT enquanto ferramenta estratégica para elevar a sua produtividade e competitividade são necessárias mudanças na estrutura organizacional e na organização do trabalho (Adler, 1992). No que diz respeito à estrutura organizacional, Castells (1996) vê a formação de redes (networks) como uma tendência inexorável na nova fase do "capitalismo informacional" (informational capitalism). Estruturas horizontais substituiriam as velhas hierarquias burocráticas verticalizadas. A cooperação entre diversos agentes, reunidos pelas networks de 
informação, permitiria a formação de estruturas facilitadoras do estabelecimento de processos de aprendizado adequados às novas tecnologias.

Em relação à organização do trabalho, os novos sistemas de produção devem ser capazes de explorar a flexibilidade permitida pela nova tecnologia e, com isso, responder, com agilidade, às demandas competitivas. Nesse caso, acredita-se que a qualidade do processo de aprendizado introjetado por uma empresa é fortemente dependente da sua organização do trabalho. Para operar essas novas estruturas e sistemas, torna-se necessária uma mão-de-obra muito mais qualificada do que a requerida por sistemas organizados em bases tayloristas/fordistas (Lazonic, 1997). Essas mudanças organizacionais levariam, em última instância, à substituição do modelo de "produção em massa" pela "produção flexível".

As mudanças organizacionais preconizadas não são triviais. Usando a terminologia Schumpeteriana, trata-se de inovações básicas e não incrementais, devido, principalmente, à sua abrangência e dificuldades quanto à implementação. Na verdade, de acordo com Ciborra e Schneider (1992), mudanças organizacionais dessa natureza dependem do estabelecimento de um processo de aprendizado diferenciado - que eles chamam de aprendizado de segunda ordem (second-order learning) -, capaz de lidar com as interdependências entre estratégia, organização e tecnologia. Esse processo levaria ao estabelecimento de rotinas organizacionais inovadoras que estruturam decisões, normas e práticas - dentro de um novo contexto formativo (formative context), definido enquanto um conjunto de compromissos mentais, comportamen ais e simbólicos compartilhados por todos os integrantes de uma organização.

A partir da diferença entre automatizar e informatizar, fica mais claro o tipo de mudança necessária para a qualificação da mão-de-obra. De acordo com Zuboff (1988), quando se adota um sistema de base microeletrônica, existem duas possibilidades de estratégias organizacionais: automatizar ou informatizar. A automatização seria simplesmente a substituição do homem pela máquina, sem mudar nada, ou quase nada, no processo produtivo. É o que procurava fazer, por exemplo, a linha de montagem fordista: substituir o esforço e a qualificação humana pela máquina. Quando se adota essa estratégia, não se depende tanto da capacidade intelectiva do trabalhador. Quando se tem uma estratégia de informatização, pelo contrário, busca-se exatamente a incorporação dessa capacidade intelectiva. Nesse caso, o operador não iria apenas responder de forma pré-programada aos comandos que aparecem em um terminal de vídeo, por exemplo. Além de fazer isso, ele seria capaz de processar esses dados, criar informações e retroalimentar o sistema produtivo a partir da sua interação com a máquina, utilizando as características dinâmicas da tecnologia e, dessa forma, explorar todo o seu potencial. Para isso, o operador teria que ter um nível de conhecimento sobre o processo significativamente maior do que nos sistemas convencionais.

As novas estruturas organizacionais, organização do trabalho e qualificação da mão-de-obra requereriam, por sua vez, práticas inovadoras de relações industriais ou, na linguagem administrativa, modernas políticas de recursos humanos. Aqui, a motivação e compromisso do trabalhador com a empresa são vistos como 
fundamentais para o estabelecimento de "organizações do trabalho de alto desempenho" (high performance work organizations). O modelo japonês é visto, geralmente, como parâmetro de boas práticas de relações industriais. Os elementos desse modelo que são mais enfatizados dizem respeito a: sistemas de recompensas que premiam a qualificação e o desempenho, treinamento intensivo e extensivo, segurança no emprego e esquemas de promoção por mérito e antiguidade. Essas práticas gerenciais seriam complementares aos novos desenhos organizacionais e adequadas ao elevado perfil de qualificação da mão-de-obra (OECD, 1996).

Outros autores, a exemplo de Locke, Kocham e Piore (1995), vão mais adiante na análise das mudanças necessárias para que vantagens competitivas sustentáveis sejam derivadas da introdução das novas tecnologias. De acordo com eles, além de aspectos organizacionais, o próprio sistema institucional de relações de trabalho - visto de uma perspectiva mais ampla do que meras práticas de administração de recursos humanos - precisaria ser modificado. A criação de um novo sistema de "relações de emprego" demandaria mudanças nas relações entre sindicatos e empresas, e seria apoiada por políticas públicas e instrumentos legais e institucionais diferentes daquelas prevalecentes na era do fordismo. A clara separação dos direitos e deveres da gerência e dos empregados - que pressupõe uma relação antagônica entre as partes - teria que ser substituída pelo compromisso mútuo com a melhoria permanente da competitividade. O papel e a voz dos trabalhadores teriam que ser ampliados, tornando-os co-responsáveis pelos destinos do empreendimento e, em contrapartida, sócios dos seus resultados (stakeholders). Importante ainda notar que, desse ponto de vista, o governo teria um papel fundamental na regulação do novo mercado de trabalho. Essa mesma visão é compartilhada pelos regulacionistas franceses ao adotarem o conceito de regime de regulação ou, mais recentemente, paradigma sociotécnico (Boyer e Durand, 1997).

Não existe, hoje, muita divergência sobre os requisitos organizacionais e institucionais para que se proceda à efetiva mudança paradigmática no mundo da produção, a partir das novas tecnologias de base microeletrônica. Pela divulgação de estudos de casos na literatura acadêmica, em publicações especializadas e mesmo na grande imprensa poder-se-ia concluir que a reconversão empresarial estaria ocorrendo em marcha acelerada, ou mesmo que o período de transição paradigmático já estaria quase encerrado. No entanto, para aqueles que acreditam que a mudança em foco não é trivial e que o processo de difusão de um complexo de novas tecnologias não é linear nem destituído de irregularidades, caberia perguntar: até que ponto essas mudanças estão realmente ocorrendo? Em outras palavras, como se encontra, na realidade, o processo de difusão das inovações organizacionais e institucionais que seriam requeridas para o aproveitamento de todo potencial da revolucionária tecnologia da informação?

Appelbaum e Batt (1994) apresentam uma revisão dos estudos de difusão de novas tecnologias organizacionais nos Estados Unidos. Apesar de ser difícil uma conclusão única a partir dos diversos surveys realizados, as autoras estimam que entre um quarto e um terço das empresas americanas realizaram algum tipo de mudança na maneira como os seus empregados são gerenciados e que cerca de um 
terço das grandes empresas possuíam programas de qualidade total. Vale notar, porém, que a força de trabalho envolvida nessas mudanças é, de acordo com os estudos revistos por Appelbaum e Batt (1994), uma fração pequena da força de trabalho total. Alguns pesquisadores estimam que, no universo das grandes empresas, apenas 5 a $10 \%$ dos empregados estariam envolvidos com as novas práticas.

Uma análise mais aprofundada dessa situação é feita por Osterman (1994), cujo survey com 694 estabelecimentos ${ }^{4}$ americanos é considerado um dos mais confiáveis. Ele encontrou, em 1992, 35\% dos estabelecimentos, com 50 ou mais empregados, utilizando algum a forma de "organização flexível do trabalho" (grupos semi-autônomos, rotação de tarefas, círculos de qualidade e Gerência pela Qualidade Total). Essas técnicas são utilizadas nas mais diferentes combinações (clusters) e, em 36\% dos casos em que mais de $50 \%$ da força de trabalho está envolvida, apenas uma das técnicas é empregada. Além disso, não prevalece uma combinação única de técnicas. Esses resultados levaram Osterman a concluir que não é possível identificar, ainda, um padrão dominante de organização que possa ser denominado "sistema de alto desempenho" (High Performance Work Systems).

A observação de que não estaria havendo, pelo menos por enquanto, o desenvolvimento de uma "única e melhor forma" de organização é compartilhada por vários autores, a exemplo de Appelbaum e Batt (1994); Locke, Kocham e Piore (1995) e Boyer e Durand (1997). Com isso, a ideia de que o "modelo japonês", baseado em qualidade total e just-in-time, estaria se difundido aceleradamente e tomando o lugar do "modelo fordista", muito em voga nos anos oitenta, estaria comprometida. Os modelos sueco - baseado na teoria sociotécnica -, alemão - com ampla participação dos trabalhadores nas decisões estratégicas das empresas - e italiano - que inspirou o conceito de "especialização flexível" - são citados como exemplos alternativos. Isso não quer dizer, no entanto, que os modelos diferenciados ou em gestação - como seria o caso americano, segundo Appelbaum e Batt (1994) - ignorem técnicas desenvolvidas ou adaptadas pelas empresas japonesas. Porém, Dosi, Giannetti e Toninelli (1992) vão além ao afirmarem que não existiria nenhuma tendência à convergência em torno de um único modelo organizacional. As evidências indicam que se anda no terreno de práticas organizacionais diferenciadas que competem no mesmo ou em diferentes espaços (Nelson, 1996).

Os resultados de Osterman (1994) são confirmados, em linhas gerais, por outros trabalhos (Kling, 1995, Ichniowski et al.). Contudo, esses mesmos autores, assim como Appelbaum e Batt (1994), chamam atenção para um ponto que é muito importante quando se trata de avaliar o impacto de mudanças gerenciais no desempenho das empresas. A implantação de novos sistemas gerenciais, ou conjunto integrado e coerente de inovações tem, em geral, levado à melhoria do desempenho. A implantação de técnicas individuais, no entanto, não está associada ao crescimento da produtividade, sendo que, em alguns casos, pode levar ao seu decréscimo.

\footnotetext{
${ }^{4}$ Osterman (1994) define um estabelecimento como um endereço comercial, o que o distingue da definição de empresa.
} 
Mesmo assim, a maioria das empresas que estão se modernizando continuam adotando uma estratégia incremental, quando se trata de inovações gerenciais. ${ }^{5}$

Essa última observação pode ser ampliada, quando se admite que inovações na organização do trabalho fazem parte de um conjunto maior, onde também estão incluídas as políticas de recursos humanos. Nesse sentido, a pesquisa de Osterman (1994) encontrou um quadro contraditório. Embora na maioria das vezes a adoção de técnicas de trabalho flexíveis seja acompanhada de sistemas de recompensas inovadores, treinamento extensivo e esforços para induzir maior compromisso da força de trabalho, outros itens constantes da "pauta japonesa" de relações de trabalho não são encontrados, a exemplo da segurança no emprego e promoção por antiguidade. Esses resultados são confirmados por Ichniowski et al. (1996) no caso americano e por diversos estudos nacionais reunidos por Locke, Kocham e Piore (1 995). Esses últimos autores confirmam também que, tanto o aparato regulador do mercado de trabalho, como as políticas nacionais de emprego não têm mudado substancialmente na direção da sua adaptação às novas realidades tecnológicas e competitivas. Por último, ainda vale observar que os estudos de difusão de inovações organizacionais geralmente vinculam a adoção das novas técnicas a determinadas estratégias competitivas. Em linhas gerais, pode-se dizer que firmas que enfrentam o novo ambiente competitivo com base em estratégias baseadas em custos e preços tendem a ser mais conservadoras nas suas práticas de organização do trabalho e de recursos humanos. Já estratégias baseadas em diferenciação pela qualidade, valor adicionado e melhores serviços geralmente impõem modernizações gerenciais mais amplas (Locke, Kocham e Piore, 1995). Da mesma forma, a pesquisa de Osterman (1994) encontrou uma correlação positiva entre estratégias de alto valor agregado (que ele chama de high-road strategy) e a adoção de práticas de trabalho flexíveis. Em resumo, as evidências sobre a difusão das inovações organizacionais capazes de explorar o potencial da IT compõem um quadro que é bastante diferente daquele pintado pelos estudos de casos baseados nas histórias de sucessos relatadas pelos gerentes e consultores que as implementaram. No mundo das empresas dos países desenvolvidos parece que ainda impera aquilo que Child e Loveridge (1990) chamaram de "conservadorismo empresarial" (organizational conservatism). Esse tipo de atitude pode ser explicado pelas consideráveis barreiras à difusão que prevalecem (Ichniowski et al, 1996).

\section{CONCLUSÕES}

As abordagens econométricas baseadas na função de produção da tradição neoclássica mostram-se insuficientes para explicar o Paradoxo de Solow. As quan-

\footnotetext{
${ }^{5}$ Muito embora os trabalhos de Osterman (1994), Appelbaum e Batt (1994), Klíng (1995) e Ichniowski et all (1996) se refiram à realidade americana, conclusões parecidas podem ser encontradas em Senker (1992), para o caso da Inglaterra e Child e Loveridge (1990) e Locke, Kocham e Piore (1995) para outros contextos. No entanto, as diferenças entre os casos nacionais não podem ser desconsideradas
} 
tificações advindas desse tipo de abordagem podem ser úteis para tentar identificar grandes tendências e a magnitude geral do problema. Mas, a teoria tradicional de crescimento econômico não consegue explicar as causas que estimulam e impedem a expansão da produtividade em um contexto de rápidas transformações tecnológicas. Sabe-se que o crescimento da produtividade e da produção é estimulado pela interação de três variáveis principais: acumulação de capital, inovação tecnológica e educação. Sabe-se, também, que, em épocas de intensas mudanças tecnológicas, a realocação de recursos produtivos e a obsolescência do "capital relativo ao conhecimento" são muito rápidas e não lineares. Em sendo assim, investimentos, tecnologia e educação devem ser vistos como forças complementares, o que torna quase que sem sentido a tarefa de tentar dividir os créditos relativos ao impacto de cada força no crescimento econômico e na produtividade a partir da função de produção agregada.

Fora do âmbito da teoria econômica convencional, no entanto, o Paradoxo poderia ser explicado enquanto uma fase de transição de paradigma tecnoeconômico, quando um novo regime tecnológico, organizacional e institucional estaria se consolidando. Esse complexo processo de transição não é linear nem suave. Ele comporta incertezas, caminhos alternativos, recessões e, portanto, comportamentos aparentemente paradoxais de variáveis macroeconômicas, como a taxa de crescimento da produtividade agregada. Essa é uma linha explicativa comungada por várias correntes teóricas a exemplo dos neo-schumpeterianos, regulacionistas ou sociólogos preocupados com os movimentos de longo prazo do capitalismo (Castells, 1996).

Na presente transição paradigmática para um regime capitaneado pela microeletrônica, ganha importância a difusão de inovações gerenciais e organizacionais, em paralelo à difusão das inovações tecnológicas. Elas são imprescindíveis para que o potencial revolucionário da tecnologia da informação possa ser transformado em aumentos de produtividade, condição necessária, mesmo que insuficiente, para a melhoria dos níveis de emprego, salários e condições de vida. O co-desenvolvimento de tecnologias, organizações e mercados pressupõe, por sua vez, mudanças institucionais e no padrão de intervenção das políticas públicas. Em sendo assim, parece lícito afirmar que as trajetórias de difusão das novas tecnologias guardam relação com os diferentes contextos sociais e institucionais encontrados.

As evidências disponíveis a respeito das mudanças microeconômicas, em curso, indicam que a constituição de um novo regime sociotécnico ainda é um processo em gestação. As mudanças, em larga escala, de estilos gerenciais, padrões organizacionais, relações de trabalho e correspondentes instituições ainda encontram barreiras consideráveis, principalmente de ordem social. Essa constante interação entre tecnologia e sociedade tende a conformar diferentes estruturas de aprendiza do que, por sua vez, condicionam o processo de difusão e mudança tecnológica. Consequentemente, não se pode pensar em mecanismos que conduzem à convergência para um único padrão de desenvolvimento tecnológico e organizacional, como se existisse uma inevitabilidade histórica.

A polêmica em torno do Paradoxo de Solow encerra algumas lições para o atual momento da economia brasileira. Primeiro, o atual estágio de difusão das novas 
tecnologias ainda apresenta oportunidades para países que se encontram mais atrasados na adoção do novo regime. Porém, não existe nenhuma razão plausível para se acreditar que as simples forças do mercado serão suficientes para que um dado país possa aproveitar essas oportunidades. Em certas circunstâncias, abertura comercial pode servir como um estímulo ao aumento da produtividade por parte das empresas submetidas ao processo de competição internacional. Mas, se o objetivo, como propõe Franco (1996), é que se estabeleça um novo "modelo de crescimento", a simples abertura não parece ser suficiente. Para além da polêmica em torno da política cambial, a abertura comercial, sem o paralelo fortalecimento da competitividade interna, pode resultar em perda de mercado das exportações, principalmente de bens industriais de alto valor agregado, como é corrente no caso brasileiro (Teixeira e Ferraz, 1997). Aumento dos investimentos, qualificação da mãode-obra e difusão, com intenso aprendizado, das novas tecnologias são os elementos básicos de uma política voltada para integrar competitivamente nossa economia no mundo globalizado. As experiências de outros países com a implementação de políticas públicas voltadas para o fortalecimento do aprendizado de tecnologias de base microeletrônica parecem ser úteis para o atual momento brasileiro.

No que se refere às inovações gerenciais, deve-se observar que a simples difusão da Gerência pela Qualidade Total e certificação pela ISO 9000, como têm sido feitas no Brasil desde o início da década de 1990, não garantem competitividade. Muito embora essas iniciativas possam representar um avanço em relação à realidade gerencial brasileira, as mudanças organizacionais necessárias são mais complexas do que a implantação de técnicas importadas. A tendência à diferenciação dos modelos gerenciais requer um esforço de adaptação e desenvolvimento de padrões alternativos, capazes de interagir com a realidade social e institucional específica. A reestruturação produtiva dos anos noventa tem sido ainda caracterizada pela execução de downsizings que, mesmo possibilitando a sobrevivência no curto prazo, podem resultar em situações de perda de mercado e desvalorização das empresas. Nesse terreno, também há muito espaço para políticas públicas que estejam preocupadas com uma real reconversão produtiva em torno das novas tecnologias já disponíveis. Afinal, o aprendizado tecnológico é um processo que acontece em um meio social e que, portanto, não pode ser entendido sem levar em consideração o contexto institucional e cultural específico.

\section{REFERÊNCIAS BIBLIOGRÁFICAS}

ABRAMOVITZ, M. (1992) “The Postwar Productivity Spurt and Slowdown Factors of Potential and Realization”, em Technology and Productivity: The Challenge for Economic Policy. Paris: Organização para a Cooperação e Desenvolvimento Econômico.

ADLER, P. (1992) “Introduction”, em ADLER, P. (org.), Technology and the Future of Work. Oxford: Oxford University Press.

APPELBAUM, E. e BATI, R. (1994) The New American Workplace. Ithaca, New York: ILR Press.

ARROW, K. (1962) “The Economic Implications of Learning by Doing”. Review of Economic Studies, vol. 29 , n. 80 . 
BAILY, M. e CHAKRABARTI, A. (1988) Innovation and the Productivity Crisis. Washington: The Brookings Institution.

BAILY, M. e GORDON, R. (1988) “The Productivity Slowdown, Measurement Issues, and the Explosion of Computer Power", Brookings Papers of Economic Activity, n. 2.

BAILY, M., BARTELSMAN, E. e HALTIWANGER, J. (1994) “ Downsizing and Productivity Growth: Myth or Reality?” Nacional Bureau of Economic Research Working Papers Series, Working paper n. 4741.

BAUMOL, W; NELSON, R. e WOLFF, E. (1994) "Introduction: The Convergence of Productivity, lts Significance and lts Varied Connotations", em BAUMOL, W; NELSON, R. e WOLFF, E. (orgs.), Convergence of Productivity: Cross-national Studies and Historical Evidence. New York: Oxford University Press.

BELL, G., CHESNAIS, F. e WIENERT, H. (1992) “Highlights of the Proceedings”, em Technology and Productivity: The Challenge for Economic Policy. Paris: Organização para a Cooperação e Desenvolvimento Econômico.

BOYER, R. e DURAND, J. (1997) After Fordism. London: Macmillan Press.

BRYNJOLFSSON, E. (1993) “ The Productivity Paradox of Information Technology”, Communications of the ACM, vol. 36, n. 12.

BRYNJOLFSSON, E. e HITT, L. (1993) “Is Information Systems Spending Productive? New Evidence and New Results”. International Conference on Information Systems, Orlando, Florida.

CASTELLS, M. (1996) The Rise of the Network Society. Oxford: Basil Blackwell

CIBORRA, C. e SCHNEIDER, L. (1992) "Transforming the Routines and Contexts of Management, Work, and Technology”, em ADLER, P. (org.), Technology and the Future of Work. Oxford: Oxford University Press.

CLEMENS, E. (1991) "Evaluation of Strategic Investments in Information Technology", Communications of the ACM, vol. 34, n. 1.

CHILD, J, e LOVERIDGE, R. (1990) Information Technology in European Services: Towards a Microelectronic Future. Oxford: Basil Blackwell.

DAVID, P. (1990) "The Dynamo and the Computer: An Historical Perspective on the Modems Productivity Paradox", American Economic Review, vol. 80, n. 2.

DOSI, G. (1985) "Technological Diffusion: The Theory and Some Methodological Suggestions for the Brazilian Case”. Brasília: IPEA (mimeo).

DOSI, G.; GIANNETTI, R.; E TONINELLI, P. (1992) Introduction: Theory and History of Technology and Business Firms: The Microeconomics of Industrial Development”, em DOSI, G.; GIANNETTI, R.; E TONINELLI P. (orgs), Techonology and Enterprise in a Historical Perspective. Oxford: Clarendon Press.

FARBEY, B.; TARGETT, D. e LAND, F. (1995) “Introduction”, em FARBEY, B.; TARGETT, D. e LAND, F. (orgs.), Hard Money - Soft Outcomes: Evaluating and Managing the IT Investment. Henley on Thames: Alfred Waller.

FLAMM, K. (1996) Mismanaged Trade? Strategic Policy and the Semiconductor Industry. Washington, D.C.: Brookings Institution Press.

FRANCO, G. (1996) “A Inserção Externa e o Desenvolvimento”, Banco Central do Brasil, mimeo. FREEEMAN, C. (1992) "The Nature of Innovation and the Evolution of the Productive System", em Technology and Productivity: The Challenge for Economic Policy. Paris: Organização para a Cooperação e Desenvolvimento Econômico.

GALLIERS, R. (1995) "Rethinking IT Evaluation in the Context of Business Systems Strategy" em FARBEY, B.; TARGETT, D. e LAND, F. (orgs.), Hard Money - Soft Outcomes: Evaluating and Managing the IT Investment. Henley on Thames: Alfred Waller

GORDON, R. (1993) “The Jobless Recovery: Does it Signal a New Era of Productivity-Led Growth?”, Brookings Papers of Economic Activity, n. 1.

ICHNIOWSKI, C.; KOCHAN, T.; LEVINE, D.; OLSON, C. E STRAUSS, G. (1996) "What Works at Work: Overview and Assessment". Industrial Relations, vol. 35, n. 3.

JOHNSON, B. (1992) "Institutional Learning”. Em LUNDVALL, B-A (ed), National Systems of Innovation: Towards a Theory of Innovation and Interactive Learning. London: Pinter Publishers. 
KLING, J. (1995) "High Performance Work Systems and Firm Performance”. Monthly Labor Review, vol. 118, n. 5.

LAZONIC, W. (1997) Organizational Learning and International Competition: The Skill-Base Hyphotesis. New York: The Jeromy Levy Economics Institute of Bart College, Working Paper n. 201.

LINDBECK, A. (1992) "Lessons from the Conference". Em Technology and Productivity: the Challenge for Economic Policy. Paris: Organização para a Cooperação e Desenvolvimento Econômico.

LOCKE, R.; KOCHAM, T. e PIORE, M. (1995) Employment Relations in a Changing World Economy. Cambridge, Massachusetts: The MIT Press.

LOVEMAN, G. (1994) “An Assessment of the Productivity Impact of Information Technologies”. Em T. ALLEN, e M. SCOTT-MORTON (orgs.), Information Technology and the Corporation of the 1990s. New York: Oxford University Press.

LUNDVALL, B-A. (1992) National Systems of Innovation: Towards a Theory of Innovation and Interactive Learning. London: Pinter Publishers.

MAIRESSE, J. e GRILICHES, Z. (1990) "Heterogeneity in Panel Data: Are There Stable Production Functions?”. Em CHAMPSAUR, P. (org.), Essays in Honor of Edmond Malivaud, vol. 3, Empirical Economics. Cambridge, Massachusetts: MIR Press.

MALERBA, F. (1992) "Learning by Firms and Incremental Technical Change". The Economic Journal, vol. 102, n. 7.

NATIONAL RESEARCH COUNCIL (1994) Information Technology in the Service Society. Washington, D.C.: National Academy Press.

NELSON, R. (1996) The Sources of Economic Growth. Cambridge, Massachusetts: Harvard University Press.

NELSON, R. e WINTER, S. (1982) An Evolutionary Theory of Economic Change. Cambridge, Massachusetts: Harvard University Press.

OECD (1996) Technology, Productivity and Job Creation. Paris: Organização para a Cooperação e Desenvolvimento Econômico

OLINER, S. (1995) "Is a Productivity Revolution Under Way in the United States?", Challenge (Special Report: The Productivity Question), vol. 38, n. 6.

OLINER, S. e SICHEL, D. (1994) “Computers and Output Growth Revisited: How Big is the Puzzle?", Brookings Papers on Economic Activity, n. 2.

OSTERMAN, P. (1994) “How Common Is Workplace Transformation and Who Adopts It?'. Industrial and Labor Relations Review. vol. 47, n. 2.

ROACH, S. (1991) "Services Under Siege - The Restructuring Imperative”, Harvard Business Review, vol. 69, n. 5 .

ROACH, S (1996) “The Hollow Ring of the Productivity Revival”, Harvard Business Review, vol. 96, n. 6.

SENKER, P. (1992) “Automation and Work in Britain". Em ADLER, P. (org.), Technology and the Future of Work. Oxford: Oxford University Press.

SOLOW, R. (1956) "A Contribution to the Theory of Economic Growth", Quarterly Journal of Economics, vol. 70, n. 1.

STRASSMAN, P. (1997) The Squandered Computer. New York: Information Economics Press.

TEIXEIRA, F. e FERRAZ, J. (1997) "Economic Instability, Market Opening and Adjustment Strategy in the Brazilian Industry". Industrial Organization and Regulation Abstracts, vol. 2, n. 16. Social Science Research Network (SSRN).

TRIPLETT, J. (1994) “Comentários e Discussão ao artigo de Oliner e Sichel”, Brookings Papers on Economic Activity, n. 2.

ZUBOFF, S. (1988) In the Age of the Smart Machine. New York: Oxford University Press. 